\title{
Le tournant dans l'enseignement des langues étrangères chez Wilhelm Viëtor dans le contexte linguistique de l'époque
}

The turning point in foreign language teaching as seen in Wilhelm Viëtor's work in the linguistic context of the time

\section{Gerda Haßler}

\section{(2) OpenEdition \\ Journals}

\section{Édition électronique}

URL : https://journals.openedition.org/dhfles/7416

DOI : $10.4000 /$ dhfles.7416

ISSN : 2221-4038

\section{Éditeur}

Société Internationale pour l'Histoire du Français Langue Étrangère ou Seconde

\section{Édition imprimée}

Date de publication : 1 décembre 2020

Pagination : 75-94

ISSN : 0992-7654

\section{Référence électronique}

Gerda Haßler, «Le tournant dans l'enseignement des langues étrangères chez Wilhelm Viëtor dans le contexte linguistique de l'époque ", Documents pour l'histoire du français langue étrangère ou seconde [En ligne], 64-65 | 2020, mis en ligne le 03 mars 2021, consulté le 26 mars 2023. URL : http:// journals.openedition.org/dhfles/7416; DOI : https://doi.org/10.4000/dhfles.7416

Ce document a été généré automatiquement le 26 mars 2023.

Tous droits réservés 


\section{Le tournant dans l'enseignement des langues étrangères chez Wilhelm Viëtor dans le contexte linguistique de l'époque}

The turning point in foreign language teaching as seen in Wilhelm Viëtor's work in the linguistic context of the time

Gerda Haßler

1 Pour son écrit polémique Der Sprachunterricht muss umkehren (1882), (L'enseignement des langues vivantes doit faire «volte-face »), Wilhelm Viëtor (1850-1918) avait choisi le sous-titre ein Beitrag zur Überbürdungsfrage von Quousque Tandem (une contribution à la question de la surcharge par Quousque Tandem), rendant hommage à l'auteur d'un écrit intitulé Die Überbürdung der Schuljugend (1882), (La surcharge de la jeunesse scolaire), publié peu de temps auparavant. Cet auteur était Friedrich Wilhelm Fricke (1810-1852) qui avait suivi la théorie pédagogique de Johann Friedrich Herbart (1776-1841), connu comme successeur d'Immanuel Kant (1727-1804) à la chaire de Königsberg et fondateur d'une pédagogie moderne, fondée sur la psychologie et une méthodologie complexe qui vise à la clarté, l'association, le système et la méthode. Fricke avait plaidé d'une manière passionnée pour une orthographe simplifiée et phonologique de l'allemand (Fricke 1877, 1880), ce qui est un autre point de rencontre avec Viëtor. Dans cette contribution, j'analyserai l'écrit polémique de Viëtor pour le contextualiser dans les courants de la linguistique de son temps (cf. Haßler 2019) et découvrir des influences possibles.

\section{Le contexte linguistique de Viëtor}

Dans la première partie de ma contribution, j'essaierai d'analyser le contexte linguistique de Viëtor. Les idées de Viëtor peuvent être principalement expliquées par le développement de la phonétique et par l'intérêt que leurs représentants portaient à l'enseignement. Bien que le préjugé de l'influence de la théorie humboldtienne sur le 
développement de la méthode orale et inductive et sur Viëtor en particulier survive encore, ce sont surtout les travaux des phonéticiens de l'époque, entre autres JeanPierre Rousselot (1846-1924), Henry Sweet (1845-1912) et Alexander Graham Bell (1847-1922) qui ont pu faire progresser cette méthode.

4 Viëtor partageait avec Rousselot l'« objet tout pratique» (Rousselot 1902:9) de la description de la prononciation qui était lié à un but de normalisation et d'unification :

Le sentiment d'unité, qui se manifeste dans tous les pays, fait naître le besoin d'un type unique pour chaque langue nationale non seulement dans l'écriture (ce qui existe déjà), mais aussi dans la prononciation (ce qui est encore loin de se réaliser complètement). D'autre part, celui qui consacre à l'étude d'une langue étrangère de longues années, a bien le droit d'exiger qu'on la lui enseigne sous sa forme la plus pure. (Rousselot $1902: 9$ )

5 «Le français, c'est donc originairement la langue de Paris ", écrit Rousselot (1902:11). Mais comme son principal moyen d'expansion a été l'écrit, il s'est laissé pénétrer par tous les patois qu'il allait remplacer. Le français ne serait pas une langue artificielle, comme l'italien ou l'allemand, imposée par de puissantes œuvres littéraires, mais la langue du roi portée par l'administration dans les provinces. "La littérature n'est venue qu'après coup " (Rousselot 1902: 11). Cette postériorité de la littérature est transposée à l'enseignement des langues et partagée par Viëtor. Bien sûr, la description exacte et l'illustration par images de l'appareil articulatoire, des positions des lèvres ou de l'élévation de la langue ne se trouvent pas chez Viëtor, mais on trouve chez lui un schéma des organes articulatoires très semblable à celui de Rousselot : 

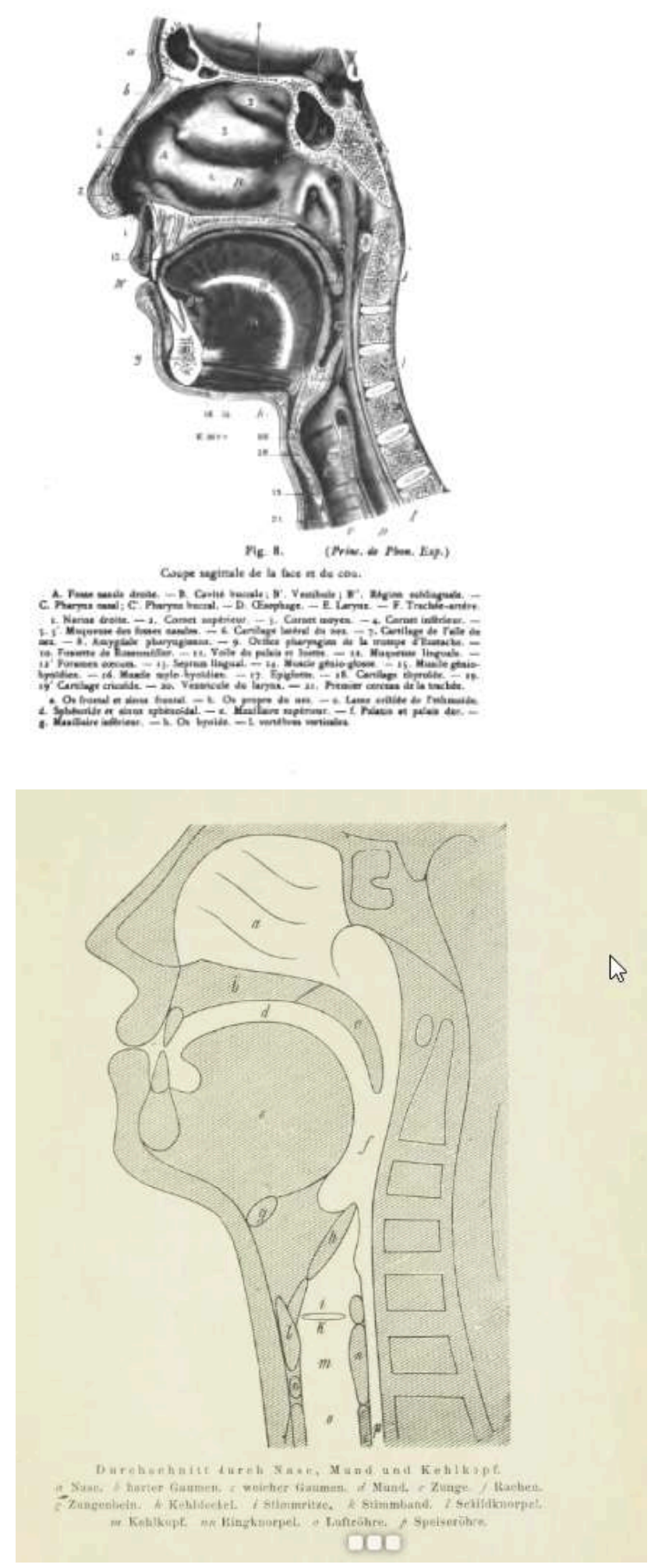

Rousselot/Laclotte 1902, 20 Viëtor 1890, p. I

6 Le premier pas vers l'apprentissage de la prononciation était, selon les phonéticiens, l'émancipation de l'épellation par lettres. Sweet a clairement décrit que la relation entre les sons et les lettres n'est pas univoque :

The first step is, to emancipate ourselves from the influence of our traditional spelling. Every one realizes that rite, write, right, wright all express exactly the same 
sound, and that ow in bow-window and to make a bow expresses two distinct sounds; but many have a difficulty in realizing that father and farther, savour and save her have exactly the same sound in educated Southern English speech. (Sweet 1892 : 2-3)

7 Pour faire apprendre une bonne prononciation de l'allemand, Viëtor a publié un manuel de lecture en écriture phonétique (Viëtor 1899/1902) qui ressemble beaucoup aux exercices que Rousselot avait intégrés dans son Précis de prononciation française. Les auteurs avaient élaboré leurs propres systèmes de transcription, mais à partir de la quatrième édition de 1906, Viëtor utilisait l'alphabet phonétique de l'Association Phonétique Internationale :

LA CIGALE ET LA FOURMI

La Cigale ayant chanté Tout l'été,

Se trouva fort dépourvue

Quand la bise fut venue.

Pas un seul petit morceau

De mouche ou de vermisseau.

Elle alla crier famine

Chez la fourmi sa voisine, La priant de lui prêter Quelque grain pour subsister Jusqu'à la saison nouvelle. Je vous paierai, lui dit-elle, Avant l'août, foi d'animal, la sigal e la furmi

la sigal eyã $€ \tilde{a} t e ́$ tu le été, sce truva fòr dépurvú kã la biza fu voenu, páz $\tilde{e}$ scel pati morsó do muє u doe vèrmisó. el ala kriyé famin eé la furmi sa vwazin, la priya doe lüi preté kelka grẽ prir subzisté juska la sezõ nuvela. joe vu père, lẅi dit_ela, avã $l$ $\dot{\text {, fwa }}$ d animal,

Rousselot/Laclotte 1902 : 196-197

\begin{tabular}{|c|c|}
\hline 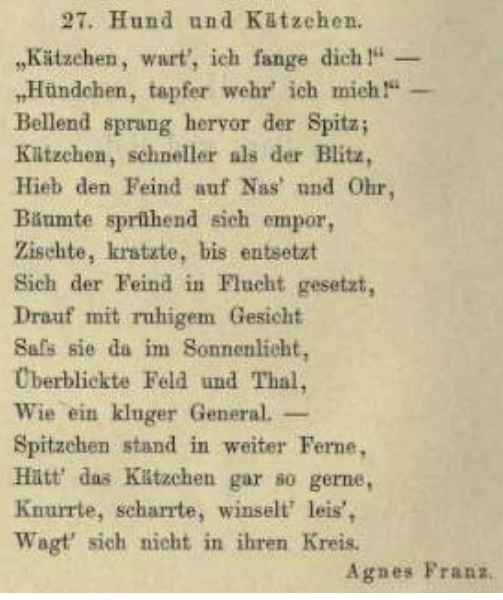 & 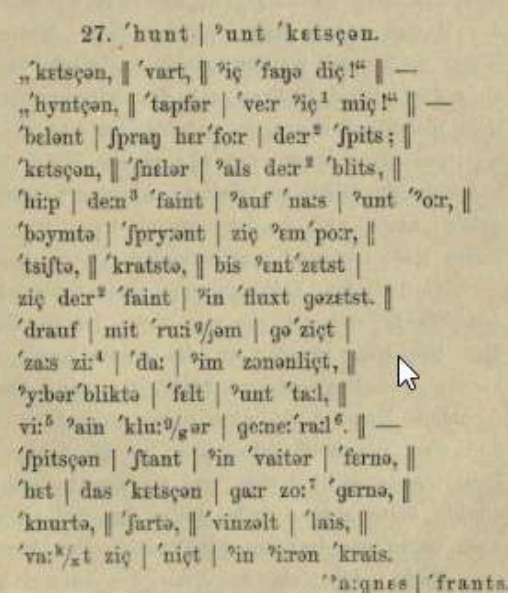 \\
\hline
\end{tabular}

Viëtor 1899/1902 : I, 82-83

L'orientation vers la langue parlée était une attitude que Viëtor avait en commun avec les phonéticiens de l'époque et qu'il leur a probablement empruntée. De plus, l'orientation vers le vocabulaire de la langue quotidienne était soutenue par les travaux en dialectologie (par exemple Georg Wenker [1852-1911], Jules Gilliéron [1854-1926], Jean Haust [1868-1946]). Il est peut-être moins évident que la linguistique historicocomparative, notamment l'école des néogrammairiens avec ses méthodes positivistes (cf. Koerner 1982), ait pu avoir une certaine influence sur le développement de la méthode orale et inductive de l'enseignement. En ce qui concerne Viëtor, sa formation linguistique était largement influencée par la linguistique historico-comparative. Cet auteur commença sa carrière par l'étude du dialecte de sa région (Viëtor 1875) et il 
continua avec des travaux sur des textes anciens qui renforcèrent son intérêt pour la phonétique (Viëtor 1876, 1884).

Un support de la méthode orale et inductive en général était certainement la conception de la langue comme une activité, provenant de la théorie linguistique de Wilhelm von Humboldt (1767-1835) (cf. Thouard 2016). Après l'analyse de l'œuvre de Viëtor, il n'est pas possible d'établir un lien direct avec cette idée, mais l'idée humboldtienne de la langue comme une energeia, c'est-à-dire une activité créatrice permanente qui ne peut pas être décrite dans un état fixé, était présente chez beaucoup d'auteurs de l'époque. Quand on suit les indications données par Viëtor lui-même, il la devait à l'orientaliste Archibald Henry Sayce (1846-1933) dont il avait probablement lu les publications lors de son séjour à l'Université de Liverpool comme professeur d'allemand. Il ne cite pas exactement l'ouvrage où il avait trouvé l'idée qu'on ne peut pas décrire le dictionnaire d'une langue parce que celle-ci est en changement constant, mais dans la grammaire assyrienne, il y a le passage suivant :

A dictionary, in the true sense of the word, is impossible: we can have only a vocabulary which is being continually enlarged and corrected. (Sayce $1872: \mathrm{V}$, voir aussi Sayce 1877)

Le concept d'une créativité linguistique qui produit continuellement de nouveaux mots en relation avec le développement des connaissances humaines était très courant à l'époque et Sayce n'avait pas besoin de l'emprunter à Humboldt. Humboldt qui avait proclamé l'identité du langage et de la pensé et, ainsi, une vision du monde déterminée par les langues particulières, se trouvait en conflit avec un enseignement des langues étrangères qui refusait de prendre en compte la langue maternelle.

11 Une autre idée, présente dans l'écrit de Viëtor et très répandue à l'époque, était la conception de la langue comme un tout organique qui désobéit à un morcellement en mots et règles grammaticales. Cette idée, elle aussi apparaît chez Humboldt, mais elle a pris des formes autonomes sous la désignation d'«organisme» chez beaucoup d'auteurs (cf. Haßler 2017) :

Elle [la langue] doit posséder à chaque instant de son existence ce qui en fait un tout. Expiration immédiate d'un être organique dans sa validité sensuelle et spirituelle, elle partage la nature de toutes les choses organiques, chaque élément n'existe en elle que par l'autre, et tout n'existe en elle que par la seule force qui pénètre le tout. (Humboldt 1903-1936 : IV,3.Cf. Haßler 1991 : 120) ${ }^{1}$

Dans une perspective vitaliste, le terme " organisme " paraît avoir été introduit par Friedrich Schlegel (1772-1829) en 1808. Il nomme la langue indienne et la langue grecque "langues organiques", "par ce qu'elles renferment un principe vivant de développement et d'accroissement, et qu'elles ont seules, si je puis m'exprimer ainsi, une végétation abondante et féconde" (Schlegel 1837: 47). Le lien avec la biologie évolutionniste paraît garantir la scientificité de la linguistique. Ce lien a produit un néologisme en linguistique, la morphologie, qui devient de plus en plus importante dans les efforts des linguistes visant à construire une théorie linguistique évolutionniste en procédant à une étude comparée systématique des langues (cf. Séguy-Duclot $2011: 9$ ).

Cette idée vitaliste de l'évolutionnisme biologique n'était pas la base de tous les ouvrages qui avaient le mot « organisme » dans leur titre ou qui prétendaient le décrire (cf. Klippi 2010). Au milieu du dix-neuvième siècle, en Allemagne, le concept d'organisme apparaît dans des descriptions de langues à but didactique, par exemple dans Karl Ferdinand Becker (1841), Anton Schmitt (1851), Josef Schraut (1852), Wilhelm 
Braubach (1853). Tout d'abord, l'emploi du concept de l'organisme ne dérive pas d'une métaphore à partir de l'organisme d'un être biologique, mais plutôt de la comparaison avec un tout composé de plusieurs parties qui forment un ensemble. Dans le livre de Braubach, destiné à être utilisé dans l'enseignement, le mot «organisme» est étroitement lié à la symétrie du système :

Si l'on parle d'organisme d'une langue, cela s'entend par soi-même que l'on doit étudier la symétrie des différents éléments de l'organisme. (Braubach 1853 : X) ${ }^{2}$

Cette position n'était plus unanimement acceptée au milieu du XIX ${ }^{\mathrm{e}}$ siècle ce que l'on peut aussi démontrer avec les écrits d'auteurs mineurs. Commençons par l'Organisme de la langue de Becker, dédié à Humboldt, dans lequel l'auteur déclare que :

L'activité de parler provient avec une nécessité intérieure de la vie organique de l'homme : l'homme parle parce qu'il pense et, en même temps, avec l'activité de penser se produit l'activité de parler. (Becker $1841: 1-2)^{3}$

La mention de Humboldt dans des ouvrages sur l'organisme ne veut pas dire que l'idée humboldtienne de la langue comme un tout, émanation d'un être organique, qui déterminerait à chaque instant les différentes parties d'une langue, ait été présente dans ces écrits. Il y avait plusieurs concepts d'organisme qui partent tous d'une comparaison avec un être organique, mais dont la nature est fort différente. Ainsi, le directeur de lycée Schraut cite le philologue Georg Curtius (1820-1885) qui compare la vie d'une langue avec celle d'un État, tous deux étant un organisme fondé sur la nationalité :

On peut comparer la vie d'une langue à celle d'un État. Ces deux éléments représentent un organisme, porté par la nationalité. (Curtius, dans Schraut 1852: $3)^{4}$

Cependant, Schraut critique cette expression métaphorique qui veut exprimer la nature du langage par une image qui ne serait pas claire et qui utiliserait une ressemblance distordue. Il suppose l'existence de deux organismes, un naturel et un imité artificiellement. Curtius s'occuperait de ce dernier, d'un organisme mécanique qui ne montrerait pas la force inhérente de l'organisme vivant d'une langue (Schraut 1852, 6). Ce qu'il reproche à la méthode comparative et à Curtius, c'est que la langue est considérée comme un ensemble créé arbitrairement, mais pas comme un organisme né d'une force de vie naturelle :

Mais c'est pour cela que, dans l'exposition de Curtius, la langue se présente à la rigueur comme un organisme arbitraire et créé artificiellement, mais vraiment pas comme un « organisme " naturel, né d'une force de vie intérieure. (Curtius, dans Schraut $1852: 9)^{5}$

Dans la définition de ce qui peut être considéré comme naturel, les opinions différaient beaucoup. Tandis que Humboldt supposait un principe créateur qui assure la connexion de son organisme comme totalité individuelle, la linguistique de la deuxième moitié du $\mathrm{XIX}^{\mathrm{e}}$ siècle, se réfère à l'organisme dans une perspective purement naturaliste.

\section{L'écrit polémique L'enseignement des langues vivantes doit faire « volte-face »}

Le pseudonyme Quousque Tandem sous lequel Viëtor avait publié la première édition de son écrit polémique était vite élucidé et, dès la seconde édition (1886), le nom de l'auteur apparaît sur le frontispice. Viëtor commence par son positionnement à l'égard 
de la surcharge des élèves qu'il déclare comme réellement existante et causée par le système scolaire lui-même. L'enseignement des langues qui constitue deux tiers des heures de cours dans les lycées est, selon Viëtor, une partie importante de cette surcharge. De plus, l'acquisition des langues qui s'opère pendant ce temps considérablement long de six ou neuf ans aboutirait à un niveau très médiocre.

19 Pour trouver les raisons de ce mauvais état de l'enseignement des langues étrangères, Viëtor invite ses lecteurs à considérer l'enseignement de la langue maternelle dans les écoles élémentaires comme une «torture inutile» («nutzlose Quälerei») que les enfants ne comprennent pas. En conséquence, cet enseignement ne peut pas former l'esprit et il n'aide pas à acquérir un allemand vivant :

Au moins une fois, à l'école maternelle, ce cours et la grammaire allemande en général sont une torture inutile. Elle n'est pas comprise ; elle ne peut donc pas avoir d'effet éducatif sur l'esprit; et personne ne peut sérieusement imaginer que les enfants ont appris leur allemand vivant grâce à elle. (Viëtor ${ }^{2} 1886$ [1882] :5) ${ }^{6}$

Viëtor déplore la confusion des sons et des lettres, ces dernières ne reposant que sur une imposition arbitraire qui n'a rien à voir avec la langue elle-même. La cible de sa critique est surtout la non-coïncidence de l'image enseignée de la langue avec la réalité de celle-ci. Ainsi, on enseignerait aux élèves qu'on ne pouvait pas prononcer les consonnes sans le soutien d'une voyelle et que seules les voyelles pouvaient former une syllabe. Cela contredirait l'usage d'un énoncé en une seule consonne, par exemple quand on chasse des poules (sch), ou aussi la formation d'une syllabe par des consonnes, quand on exige le silence (bst) :

Cette confusion désastreuse de l'écriture et du langage est inoculée dans la langue de l'enfant. Malheur à lui s'il ne sait pas que les voyelles a, e, i, o, u, y, et les lettres restantes, « ou les sons » sont des consonnes! Mais maintenant, demandez à l'élève, ou même au professeur, la raison de cette division! «Vous ne pouvez pas prononcer les consonnes pour elles seules (que faites-vous si vous effrayez les poulets avec un "Sch»?), seules les voyelles peuvent former une syllabe (et pourtant l'enfant apprend : «Bst ! qui vient là silencieux et muet?») - en bref, vous entendez une absurdité phonétique qui n'a d'égale que les absurdités faites à l'école avec le mot « syllabe ». (Viëtor $\left.{ }^{2} 1886[1882]: 3\right)^{7}$

21 L'orientation selon l'écriture mène au maintien de la distinction des diphthongues $e i$ et ai [ăi : āi] et de la prononciation sonore des occlusives $b, d, g$ à la fin d'un mot qui, en réalité, se sont perdues depuis longtemps. De plus, l'enseignement erroné des maîtres aurait mené à la prononciation longue de la voyelle dans l'article das, en opposition à la conjonction dass. Pour remédier à cette situation, Viëtor se réclame d'August Schleicher (1821-1868) qui exige que chacun parle de manière spontanée la langue écrite commune et la langue de conversation soutenue, ce bijou inestimable :

Certes, il est préférable (et c'est maintenant la fin de la chanson dans son ensemble) que chacun parle «la langue écrite et le langage familier supérieur qui nous sont communs ", " ce joyau inestimable ", " à mesure que son bec a grandi ». C'est ce que Schleicher lui-même demande. (Die deutsche Sprache. 4. Aufl. v. J. Schmidt, Stuttgart, Cotta 1879) [...] (Viëtor 1886 [1882] : 4) ${ }^{8}$

22 Comme je le montrerai dans la troisième partie de ma contribution, Schleicher n'est pas seulement la première référence dans l'écrit polémique de Viëtor, mais il a élaboré une théorie linguistique dont on peut déduire les prémisses linguistiques de Viëtor.

Viëtor critique l'enseignement de la grammaire suivant les parties du discours, adopté de la langue latine et transposé à la langue allemande qui ne connaît plus de distinction 
entre substantifs forts et faibles. La grammaire souffrirait plutôt de la disparition d'anciennes terminaisons et de l'infiltration de mots étrangers dont « un ami sage de notre langue ne pourrait que se réjouir » (« über welche sich jeder einsichtige Freund unserer Sprache nur freuen kann » (Viëtor 1886 [1882] : 5).

L'application de la doctrine fondamentalement fausse de l'écriture à l'enseignement des langues étrangères donne, selon Viëtor, de mauvais résultats. Il se réfère à l'orientaliste britannique Archibald Henry Sayce pour affirmer que la langue consiste en sons et pas en lettres. C'est à lui qu'il doit l'idée qui nous paraît très humboldtienne que la langue se forme par l'activité inconsciente d'un tout et que la vie d'une société est en changement et en évolutions perpétuelles (Viëtor 1886 [1882] : 5-6). Par conséquent, on ne peut pas enfermer la grammaire d'une langue dans un certain nombre de règles fixes qui, une fois formulées par le grammairien, seraient comme les lois des Mèdes et des Persans. Tout au contraire, la grammaire est ce que le peuple en fait; ce qui était à la mode hier, est oublié aujourd'hui, et ce qui est juste aujourd'hui sera faux demain (Viëtor 1886 [1882] : 6).

Selon Viëtor, on ne parlera jamais une langue en apprenant de longues listes de mots et on ne comprendra jamais une langue en ayant simplement appris toutes les règles de sa grammaire. Son argumentation est en opposition constante avec le principe formel (" das formale Prinzip », Viëtor 1886 [1882]: 6) qu'il considère comme nuisible pour l'apprentissage d'une langue. Il critique l'enseignement de la prononciation après celui de l'écriture et selon des manuels dépassés, comme pour l'anglais celui de Walker (1791), ou encore la prononciation des consonnes du français selon le dialecte du pays natal (Viëtor 1886[1882] : 9 : chai bas gonfianze) et l'accentuation de la dernière syllabe d'un mot selon l'accent lexical allemand.

26 L'introduction de la flexion nominale est critiquée par Viëtor (1886 [1882] : 13) qui la considère comme un aveuglement dû à une croyance irréfléchie en la tradition ("Verblendung gedankenloser Traditionsgläubigkeit»). Les substantifs français n'ont plus de cas formel et le pluriel ne se marque pas dans la langue parlée, sauf dans la liaison où il est désigné par un $[z]$ sonore. L'orientation de la description vers la langue parlée se montre aussi dans l'affirmation que la forme féminine des adjectifs ne se forme pas par l'ajout de la voyelle -e. Au contraire, cette forme se marquerait par un changement de la consonne terminale ou bien elle ne se marque pas. De plus, la conjugaison française serait tout à fait différente de sa description dans les grammaires. Les formes du singulier ont perdu tout marquage de personne et, au pluriel, la troisième personne coïncide avec celles du singulier.

Viëtor constate qu'il y a beaucoup de débris issus d'un temps reculé que le fleuve de la langue emporte avec lui et il ne serait pas possible de vouloir expliquer tout cela à partir de la langue contemporaine. Ces écuries d'Augias de règles, groupes et lois phonétiques seraient donc à nettoyer. La transposition de la grammaire gréco-latine aurait peu de justification pour la syntaxe de l'anglais et du français, et aucune pour la flexion parce que la forme et le sens sont indivisibles, comme le corps et l'âme; tous les deux forment la langue et l'écriture n'est que leur vêtement ${ }^{9}$.

Dans la partie sur la méthodologie de l'enseignement, Viëtor commence par la question de savoir comment procèderait un menuisier s'il voulait enseigner son métier à un apprenti selon la méthode de la grammaire de Donat. Il lui montrerait d'abord des modèles ou des pièces de bois et il les classifierait selon leur lourdeur, dureté, couleur, 
etc. Ensuite, il classifierait les outils selon les parties constituantes en des groupes et sous-groupes. Il continuerait cet enseignement jusqu'à ce que l'apprenti sache énumérer toutes les classes et toutes les parties constituantes, sans qu'il n'ait jamais travaillé le bois au couteau ou foré un trou à l'aide d'un foreur.

La méthode de l'enseignement des langues ne serait pas très loin de cette méthode de Donat parce que les professeurs font apprendre des mots et des règles de grammaire. Viëtor se réfère encore une fois à Sayce pour répéter son axiome «La langue ne consiste pas en mots » (« Die Sprache besteht nicht aus Wörtern » (Viëtor 1886 [1882] : 20). En ce qui concerne les règles grammaticales, Viëtor (1886 [1882] : 22) plaide pour la voie inductive de leur acquisition qui permet de réveiller l'activité des élèves. L'obligation de Viëtor envers Sayce est évidente dans son écrit polémique. Il cite, entre autres, une page entière de cet auteur où il exprime son scepticisme à l'égard de l'apprentissage de langues anciennes, limité à l'apprentissage de mots et de règles et qui ne vise pas à penser dans une langue étrangère. Pour lui, une langue consiste surtout en des sons, prononcés par des êtres humains et remplis de sens.

30 L'écrit polémique L'enseignement des langues vivantes doit faire "volte-face» est souvent considéré comme acte de naissance de la méthode directe dans l'enseignement des langues. On lui attribue l'idée de l'usage exclusif de la langue étrangère et de l'exclusion absolue de la langue maternelle. Cette idée inclut l'enseignement inductif de la grammaire. En considérant que Viëtor avait inventé la méthode directe, on méconnaît qu'il y eut des écrits antérieurs qui avaient proposé d'enseigner les langues par des méthodes proches et des précurseurs de la voie orale et inductive. Ainsi, par exemple, Gottlieb Heness (1884), dans son Leitfaden für den Unterricht der Deutschen Sprache («Guide pour l'enseignement de la langue allemande»), avait souligné la communication naturelle comme activité centrale de l'enseignement de la langue, et Claude Marcel (1867), dans L'étude des langues ramenée à ses véritables principes, ou l'art de penser dans une langue étrangère avait décrit l'apprentissage d'une langue par la compréhension orale et écrite. Le professeur de langue François Gouin publia en 1880 L'art d'enseigner et d'étudier les langues où il propageait l'enseignement des langues étrangères sans règles et sans traduction, mais à l'aide de visualisations et d'activités ludiques en relation avec des situations quotidiennes. Il est difficile d'établir des lignes de filiation des idées, mais on peut certainement dire que l'enseignement des langues à partir de la communication orale était très répandue pendant le dernier tiers du XIX ${ }^{e}$ siècle ( $c f$. Besse 1980, 2012, Christ 1990). du génie de la nation se trouve remplacé chez Schleicher par la définition de la langue comme organisme naturel dont l'évolution se retrace à partir d'une première cellule linguistique indifférenciée (Schleicher [1860] 1869, 1863, 1868, cf. Schlanger 1995 [1971] : 129). langage, publié en 1863 et, dans sa traduction française, en 1868. Il s'agit d'une lettre ouverte à Ernst Haeckel (1834-1919) dans laquelle il applique à la vie des langues la notion de "lutte pour la vie » et il suggère le transfert des catégories des sciences naturelles à la linguistique. Il déclare nettement que la glottique ou science du langage est une science naturelle : 
Les langues sont des organismes naturels qui, en dehors de toute volonté humaine et suivant les lois déterminées, naissent, croissent, se développent, vieillissent et meurent; elles manifestent donc, elles aussi, cette série de phénomènes qu'on comprend habituellement sous le nom de vie. La glottique ou science du langage est par suite une science naturelle; sa méthode est d'une manière générale la même que celle des autres sciences naturelles. (Schleicher 1980 [1863] : 61-62])

34 (Schleicher [1860] 1869). Dans ce livre, Schleicher s'oppose nettement à l'enseignement de la grammaire pratiqué dans les écoles qui produirait une horreur de la grammaire, chez les élèves, et qui ne mènerait pas à la connaissance de l'organisme d'une langue. Il nomme la mémorisation de formes effectuée à l'école en utilisant le mot allemand composé «Jugendlustverderber » qui signifie " corrupteur des plaisirs de la jeunesse » et il lui dénie la capacité d'enseigner le génie de la langue. Ce génie qui consiste en l'organisme de la langue, ne peut pas être acquis, faute d'un enseignement adéquat et à cause du manque de livres sur le langage compréhensibles pour un grand public:

De la nature de la langue, de ses formes, de sa parenté, etc. on ne sait généralement rien; celui qui entend parler de la construction du langage et de sa représentation scientifique, de la grammaire, se détourne en général avec plaisir du souvenir éveillé par elle des temps pénibles où il fallait mémoriser j'aime, tu aimes, mensa, mensae, týpto, týpteis et autres corrupteurs des plaisirs de la jeunesse, il se réjouit de n'avoir plus rien à faire avec une matière aussi sèche et plaint profondément l'homme qui a fait de la « grammaire » sa tâche exclusive dans la vie.

Cependant, dans la manière dont l'enseignement des langues a été dispensé jusqu'à présent presque universellement, cette horreur de la grammaire, qui fait que l'on sait si peu de choses sur la nature de la langue et que l'on a une vision si insuffisante de son organisme, est en partie la conséquence de l'enseignement scolaire habituel; la raison est aussi en partie à chercher dans le fait qu'il n'y a pas de livres sur les questions linguistiques qui soient compréhensibles. (Schleicher [1860] $1869: 3-4)^{10}$

Cet organisme de la langue est conçu comme un organisme naturel, comparable aux plantes et aux animaux. Schleicher utilise plusieurs métaphores dont la vie de la langue qui reflète le plus complètement son concept. La formation d'une langue est pour lui une activité de l'homme qui précède l'histoire :

La formation du langage et l'histoire sont deux activités distinctes de l'homme, deux révélations de sa nature qui n'ont jamais lieu en même temps, mais dont la première précède toujours la seconde. (Schleicher [1860] $1869: 35)^{11}$

Avec le début de l'histoire, la décadence de la langue commence et se poursuit pendant toute l'histoire :

La vie des langues se divise donc principalement en deux périodes totalement distinctes: l'histoire du développement des langues: période préhistorique, et l'histoire du déclin de la forme linguistique : période historique. (Schleicher [1860] $1869: 37)^{12}$

\section{Conclusion}

La formation linguistique de Viëtor a été imprégnée par la linguistique historicocomparative traditionnelle, dans le cadre de laquelle il a écrit ses premiers écrits sur la 
langue. L'accent mis ensuite sur la langue parlée et la primauté accordée à celle-ci dans l'enseignement des langues étrangères ont été influencés par les phonéticiens de l'époque, dont il partageait les vues. Sa conception de l'organisme naturel du langage remonte apparemment principalement à Schleicher, qui avait critiqué l'enseignement des langues étrangères modernes de la même manière que Viëtor. Ce serait, bien sûr, une exagération que d'attribuer la parallélisation de Schleicher entre la vie des hommes et la vie des langues à Viëtor qui avait un but plus modeste à savoir la réforme de l'enseignement des langues. Mais il partage avec Schleicher la conception vitaliste et holistique de la langue qui cherche à expliquer la forme et la fonction à partir du son.

\section{BIBLIOGRAPHIE}

\section{Sources primaires}

BECKER, Karl Ferdinand (1841). Organism der Sprache. Frankfurt a.M. : G. F. Kettembeil. BRAUBACH, Wilhelm (1853). Grammatik des Styls und Organismus der Sprache. Für Schule und Wissenschaft mit pädagogisch-practischen Zugaben für den Gebrauch des Lehrers. Gießen : Ferbersche Universitätsbuchhandlung.

FRICKE, Friedrich Wilhelm (1877). Die Orthographie nach den im Bau der deutschen Sprache liegenden Gesetzen in wissenschaftlicher, paedagogischer und praktischer Beziehung. Bremen : Küthmann. FRICKE, Friedrich Wilhelm (1880). Die Reichsorthographie : zur Orientierung in dem Streite über Möglichkeit und Nützlichkeit einer einfachen Rechtschreibung. Schellenberg : Wiesbaden.

FRICKE, Friedrich Wilhelm (1882). Die Ueberbürdung der Schuljugend : ein Mahnwort an Eltern, Lehrer und Jugendfreunde der gesammten deutschen Nation. Berlin : Hofmann.

GoUIN, François (1880). Exposé d'une nouvelle méthode linguistique. L'art d'enseigner et d'étudier les langues. Paris : Fischbacher.

HENESS, Gottlieb (1884). Leitfaden für den Unterricht der Deutschen Sprache ohne Sprachlehre und Wörterbuch, mit einer englischen Einleitung über die Lehrmethode der Schule moderner Sprachen in Boston. New York : H. Holt.

HUMBоLDT, Wilhelm von (1903-1936). Wilhelm von Humboldts gesammelte Schriften, édité par Königlich Preußische Akademie der Wissenschaften. 17 vols. Berlin : B. Behr's Verlag.

MARCEL, Claude (1867). L'étude des langues ramenée à ses véritables principes, ou l'art de penser dans une langue étrangère. Paris : Borrani.

ROUSSELOT, Jean-Pierre \& LACLOTTE, Fauste (1902). Précis de prononciation française. Paris, Leipzig : Welter.

SAYCE, Archibald Henry (1872). An Assyrian grammar foe comparative purposes. London : Trübner \& Co. 
SAYCE, Archibald Henry (1877). Lectures upon the Assyrian language, and syllabary; delivered to the students of the archaic classes. London : Samuel Bagster and sons.

SCHLEGEL, Friedrich von (1837). Über die Sprache und Weisheit der Indier, trad. française Adolphe Mazure, Essai sur la langue et la philosophie des Indiens. Paris : Parent-Desbarres.

SCHLEICHER, August ([1860] 1869). Die Deutsche Sprache. Zweite verbesserte und vermehrte Auflage. Stuttgart : J. G. Cotta.

SCHLEICHER, August (1863). Die darwinsche Theorie und die Sprachwissenschaft. Weimar : Böhlau.

SCHLEICHER, August (1868). La théorie de Darwin et la science du langage, trad. française de M. de Pommayrol, avec une préface de Michel Bréal. Paris : A. Franck.

SCHLEICHER, August ([1863] 1980). La théorie de Darwin et la science du langage. Évolutionnisme et linguistique, édité par P. Tort. Paris : Vrin.

SCHмIтT, Anton (1851). Organismus der Lateinischen Sprache, oder : Darstellung des römischen Volkes in seinen Sprachformen. Mainz : Wirth Sohn.

SCHRAUT, Josef (1852). Zum Organismus der Sprache mit besonderer Rücksicht auf das Griechische.

Rastatt : W. Mayer.

SWEET, Henry (1892). A primer of phonetics. Oxford : Clarendon Press.

VIËTOR, Wilhelm (1875). Die Rheinfränkische Umgangssprache in und um Nassau. Wiesbaden : Niedner. VIËTOR, Wilhelm (1876). Die Handschriften der Geste des Loherains. Halle : Lippert.

VIËTOR, Wilhelm (1884). Elemente der Phonetik und Orthoepie des Deutschen, Englischen und Französischen mit Rücksicht auf die Bedürfnisse der Lehrpraxis. Heilbronn : s.l.

VIËTOR, Wilhelm ([1882] $\left.{ }^{2} 1886\right)$. Der Sprachunterricht muss umkehren! Ein Beitrag zur Überbürdungsfrage von Quousque Tandem. Zweite um ein Vorwort vermehrte Auflage. Heilbronn : Henninger.

VIËTOR, Wilhelm (1890). Die Aussprache des Schriftdeutschen, mit dem Wörterverzeichnis für die deutsche Rechtschreibung zum Gebrauch in den Preussischen Schulen in phonetischer Umschrift sowie phonetischen Texten. 2., umgearbeitete Auflage. Leipzig : Reisland.

VIËTOR, Wilhelm (t1:1899/t2:1902). Deutsches Lesebuch in Lautschrift (Zugleich in der amtlichen Schreibung). Als Hülfsbuch zur Erwerbung einer Mustergültigen Aussprache. 2 Bde. Leipzig : Teubner.

\section{Sources secondaires}

BESSE, Henri (1980). Polémique en didactique : du renouveau en question. Paris : CLE international. BESSE, Henri (2012). « Éléments pour une 'archéologie' de la méthode directe ». Documents pour l'histoire du français langue étrangère ou seconde, 49, 11-30.

CHRIST, Herbert (1990). « Pour et contre la méthode directe : Les débats au sein de l'Association Allemande des Professeurs de Langues Vivantes entre 1886 et $1914 »$. Études de Linguistique Appliquée, 90, 9-22.

HASSLER, Gerda (2017). « Les métaphores physiologiques comme désignations de concepts linguistiques dans la philologie romane de la deuxième moitié du XIX ${ }^{\mathrm{e}}$ et de la première moitié du XX ${ }^{\mathrm{e}}$ siècle ». In Anne-Marie Chabrolle-Cerretini (dir.), Paradigmes et concepts pour une histoire de la linguistique romande. Limoges : Lambert-Lucas. 85-96. 
HASSLER, Gerda (2019). « 'La enseñanza de idiomas debe cambiar'. El pensamiento metodológico de Wilhem Viëtor y su trasfondo lingüístico ». Boletín de la Sociedad Española de Historiografía Lingüística (BSEHL), 13, 25-40.

KLIPPI, Carita (2010). La vie du langage : La linguistique dynamique en France de 1864 à 1916. Lyon : ENS Éditions.

KOERNER, E.F. Konrad (1982). « The neogrammarian doctrine : breakthrough or extension of the Schleicherian paradigm ». In J. Peter Maher, Allan R. Bomhard \& E.F.K. Koerner (dir.), Papers from the Third International Conference on Historical Linguistics, Hamburg, August 22-26 1977. Amsterdam/ Philadelphie : John Benjamins, 129-152.

SCHLANGER, Judith ([1971] 1995). Les métamorphoses de l'organisme. Paris : L'Harmattan.

SÉGUY-DUCLOT, Alain (2011). Recherches sur le langage. Paris : Vrin.

THOUARD, Denis (2016). Et toute langue est étrangère : le projet de Humboldt. Paris : Encre marine.

\section{NOTES}

1. [...] sie [die Sprache] muß in jedem Augenblick ihres Daseyns dasjenige besitzen, was sie zu einem Ganzen macht. Unmittelbarer Aushauch eines organischen Wesens in dessen sinnlicher und geistiger Geltung, theilt sie darin die Natur alles Organischen, dass Jedes in ihr nur durch das Andre, und Alles nur durch die eine, das Ganze durchdringende Kraft besteht.

2. Spricht man von Organismus der Sprache, so sollte sich von selbst verstehen, daß man auch der den einzelnen Gliedern der Organismus eigenen Symmetrie nachgehen muss.

3. Die Verrichtung des Sprechens geht mit einer inneren Nothwendigkeit aus dem organischen Leben des Menschen hervor: denn der Mensch spricht, weil er denkt; und mit der Verrichtung des Denkens ist zugleich die Verrichtung des Sprechens gegeben.

4. Man kann das Leben einer Sprache mit dem eines Staates vergleichen. Beide stellen in sich einen von einer Nationalität getragenen Organismus dar.

5. Aber darum steht auch in Curtius Darstellung die Sprache höchstens als ein willkürlich erkünstelter, aber wahrhaftig nicht als ein naturwüchsiger, aus innerem Lebenstrieb entsprossener « Organismus » da.

6. Mindestens einmal, in der Vorschule, ist diese und überhaupt die deutsche Grammatik eine nutzlose Quälerei. Verstanden wird sie ja nicht; geistbildend kann sie daher nicht wirken; und dass die Kinder daraus ihr lebendiges Deutsch lernten, wird wohl auch niemand im Ernste sich einbilden.

7. Diese verhängnisvolle Verwechslung von Schrift und Sprache wird dem Kind mit der Sprache eingeimpft. Wehe ihm, wenn es nicht wissen sollte, dass a, e, i, o, u, y Vokale, die übrigen Buchstaben, « oder Laute » Konsonanten sind! Nun frage man aber den Schüler, oder auch den Lehrer, nach dem Grund dieser Einteilung! « Man kann die Konsonanten nicht für sich allein aussprechen » (was thut man denn, wenn man die Hühner mit «Sch » verscheucht?), « Nur die Vokale können eine Silbe bilden» (und doch lernt das Kind: «Bst! Wer kommt da still und stumm?») - Kurz, man bekommt einen Lautlichen Unsinn zu hören, dem nur der mit dem Wort «Silbe » in der Schule getriebene Unfug die Stange zu halten vermag.

8. Da ist es freilich besser (und das ist jetzt doch im ganzen das Ende vom Lied), wenn jeder « die uns allen gemeinsame Schriftsprache und höhere Umgangssprache », " dieses unschätzbare Kleinod », spricht, "wie ihm der Schnabel gewachsen ist ». So verlangt es kein Geringerer als Schleicher. 
9. Form und Sinn sind unzertrennlich, wie Leib und Seele; beide bilden die Sprache, und deren Kleid nur ist die Schrift. (Viëtor 1886 [1882] : 6).

10. Vom Wesen der Sprache, ihren Formen, Sippen u.s.f. weiß man aber in der Regel so viel als gar nichts; wer vom Bau der Sprache und von der wissenschaftlichen Darstellung derselben, von Grammatik, hört, wendet sich in der Regel von der dadurch geweckten Erinnerung an die qualvollen Zeiten, als j'aime, tu aimes, mensa, mensae, týpto, týpteis und andere Jugendlustverderber memoriert werden mußten, gerne wieder ab, freut sich mit dergleichen trocknem Kram nichts mehr zu schaffen zu haben und bedauert von Herzen den Mann, der «Grammatik » sich zur ausschließlichen Lebensaufgabe gemacht hat.

In der Art und Weise, wie bis jetzt der Sprachunterricht fast allgemein erteilt wird, liegt allerdings eine Berechtigung dieses gelinden Horrors vor Grammatik, daß man vom Wesen der Sprache so wenig kennt, vom Organismus derselben so mangelhafte Anschauungen hat, ist theils die Folge des üblichen Schulunterrichts, theils ist aber auch der Grund darin zu suchen, daß es an allgemein verständlichen Büchern über sprachliche Dinge noch so gut als völlig gebricht.

11. Sprachbildung und Geschichte sind sich ablösende Thätigkeiten des Menschen, zwei Offenbarungsweisen seines Wesens, die nie zugleich stattfinden, sondern von denen stäts die erstere der zweiten vorausgeht.

12. Das Leben der Sprache zerfällt also vor allem in zwei völlig gesonderte Perioden : in die Entwicklungsgeschichte der Sprache : vorhistorische Periode, und in die Geschichte des Verfalles der sprachlichen Form : historische Periode.

\section{RÉSUMÉS}

L'écrit polémique de Wilhelm Viëtor (1850-1918) Der Sprachunterricht muss umkehren (L'enseignement des langues vivantes doit faire « volte-face » 1882) est souvent considéré comme acte de naissance de la méthode directe dans l'enseignement des langues. On lui attribue l'idée de l'usage exclusif de la langue étrangère et l'exclusion absolue de la langue maternelle. Cette idée inclut l'enseignement inductif de la grammaire. En considérant que Viëtor avait inventé la méthode directe, on méconnaît qu'il y avait des écrits antérieurs qui avaient proposé d'enseigner les langues par des méthodes proches, précurseurs de la voie directe. Dans son écrit polémique, il cite plusieurs linguistes, sans toutefois analyser leurs théories. Il témoigna une haute estime au linguiste naturaliste August Schleicher (1821-1868) qu'il considérait comme une autorité importante. La conception de la langue comme un être organique soumis aux lois de l'évolution était compatible avec la voie inductive de l'apprentissage d'une langue.

Wilhelm Viëtor's polemical pamphlet (1850-1918) Der Sprachunterricht muss umkehren (Foreign language teaching has to change 1882) is considered by many manuals of didactics as a founding document of the direct method in foreign language teaching. The idea of the exclusive use of the foreign language and of the absolute exclusion of the native language, which includes the inductive teaching of grammar, is attributed to Viëtor. In this article, I analyse Viëtor's article, contextualise it in the tendencies in linguistics of his time and discover possible influences. Furthermore, I focus on the influence of August Schleicher (1821-1868) on Viëtor's concept of language. In the last part of the paper I try to analyse Viëtor's linguistic context. Viëtor's ideas can mainly be explained by the development of phonetics and the interest of its protagonists in language teaching 
INDEX

Keywords : Wilhelm Viëtor (1850-1918), language teaching, phonetics, direct method, organism of language

Mots-clés : Wilhelm Viëtor (1850-1918), enseignement de langues, phonétique, méthode directe, organisme de la langue

\section{AUTEUR}

GERDA HASSLER

Université de Potsdam - hassler@uni-potsdam.de 